Purification of carbon nanotubes through an electric field near the arranged microelectrodes

This article has been downloaded from IOPscience. Please scroll down to see the full text article.

2007 Nanotechnology 18115602

(http://iopscience.iop.org/0957-4484/18/11/115602)

View the table of contents for this issue, or go to the journal homepage for more

Download details:

IP Address: 143.248.103.97

The article was downloaded on 19/04/2011 at 08:39

Please note that terms and conditions apply. 


\title{
Purification of carbon nanotubes through an electric field near the arranged microelectrodes
}

\author{
Hyung Cheoul Shim ${ }^{1}$, Hyung Woo Lee ${ }^{2}$, Sujin Yeom ${ }^{1}$, \\ Yoon Keun Kwak ${ }^{1}$, Seung S Lee ${ }^{1}$ and Soo Hyun Kim ${ }^{1}$ \\ ${ }^{1}$ School of Mechanical, Aerospace and Systems Engineering, Department of Mechanical \\ Engineering, Korea Advanced Institute of Science and Technology (KAIST), 373-1, \\ Guseong-dong, Yuseong-gu, Daejeon 305-701, Republic of Korea \\ ${ }^{2}$ Massachusetts Institute of Technology (MIT), Micro and Nano Systems Laboratory, \\ 77 Massachusetts Avenue, Room 5-008, Cambridge, MA 02139, USA
}

E-mail: soohyun@kaist.ac.kr

Received 10 October 2006, in final form 11 December 2006

Published 7 February 2007

Online at stacks.iop.org/Nano/18/115602

\begin{abstract}
In this work, we attempt to purify multi-walled carbon nanotubes (MWNTs) using electrophoresis induced by the application of an AC electric field to a set of microelectrodes in a microliquid channel. This purifying method is different from conventional methods based on chemical processes. It was observed that most of the MWNTs could pass along the microliquid channel without attaching to the electrode under specific conditions of $1 \mathrm{kHz}$, at $0.2 V_{\mathrm{rms}} \mu \mathrm{m}^{-1}$. On the other hand, the majority of the carbon impurities attached to the electrodes under identical conditions. Field emission scanning electron microscopy (FESEM) images and Raman spectra confirm that this condition is beneficial for removing carbon impurities. The proposed approach has potential applicability in the development of microdevices that can simultaneously perform the purification and fabrication of MWNTs.
\end{abstract}

(Some figures in this article are in colour only in the electronic version)

\section{Introduction}

Since their discovery in 1991, carbon nanotubes (CNTs) have drawn considerable attention due to their unique physical properties and their potential use in various applications [1-5]. The beneficial characteristics of CNTs include their high mechanical strength and unusual electronic properties, making them suitable for field emission displays, their capability to store a large amount of hydrogen, a high modulus, and structural diversities that make bandgap engineering possible [6].

However, significant problems remain related to CNTs, including realizing practical applications for their use as well as those related to their mass production. In particular, it is difficult to control CNTs due to their nano-level size. Furthermore, they occasionally display irregular mechanical or electrical properties, as well as impurities that are inevitably included when a synthetic process is not used in the manufacture of CNTs. These impurities include fullerenes, amorphous carbon, etc, and they act as defects in the development and research of CNTs. Studies on the purification of CNTs have occupied a considerable portion of the research field related to CNTs, and a variety of methods to purify CNTs have been reported including chemical oxidation [7], thermal oxidation [8-10], filtration [11], and chromatography [12, 13]. Among these methods, chemical or thermal oxidation processes are simple but can potentially result in the destruction or transformation of CNTs. Chromatography, meanwhile, has a limited practical applicability because of the limited solubility of CNTs.

In the present study, we have attempted to purify CNTs through an approach involving the application of an electric field. The proposed method is simple, fast and nondestructive. To this end, we fabricated a set of microelectrodes in a microliquid channel, and attempted to identify specific purifying conditions through adjustment of the voltage and 


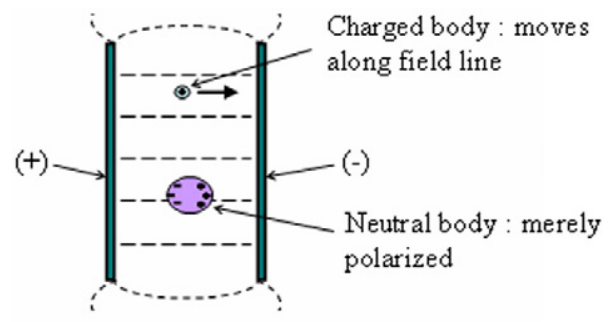

Uniform electric field

Figure 1. The phenomenon of electrophoresis.
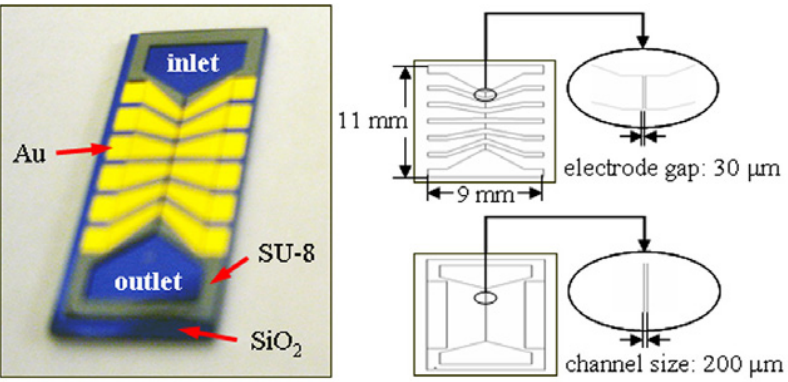

Figure 2. Specifications of the purifying device.

frequency. In addition, experimental results were assessed with field emission scanning electron microscopy (FESEM) images and Raman spectroscopy.

\section{Apparatus and theory}

\subsection{Background theory}

Dielectrophoresis or electrophoresis can be achieved by applying an alternating current (AC) or direct current (DC) electric field between electrodes. Dielectrophoresis is quite different from electrophoresis. Dielectrophoresis is the motion of neutral particles caused by a polarization effect or dipole in a nonuniform electric field. On the other hand, electrophoresis is caused by electrostatic force, which is generated by applying an $\mathrm{AC}$ or DC electric field between an electrode and a charged body. Electrophoresis can be achieved regardless of the electric field's uniformity.

Figure 1 shows the motion of neutral and charged particles in a uniform electric field, and it also describes the general aspects of electrophoresis. A charged particle is pulled along the field lines towards an electrode carrying a charge opposite to that of the particle. In the same field, a neutral body is merely polarized. The result may produce a torque, but not a net translational force, without which the body as a whole will not move towards either electrode. Electrophoresis can be appreciable even when the free charge per unit weight of the particle is quite small. It is different from dielectrophoresis in that it does not depend upon the particle volume, but rather upon the free charge on the particle [14]. Therefore, it is possible to purify the CNTs using an electric field by using the difference of motion between CNTs, a neutral body in a physical sense and carbon impurities as charged particles.

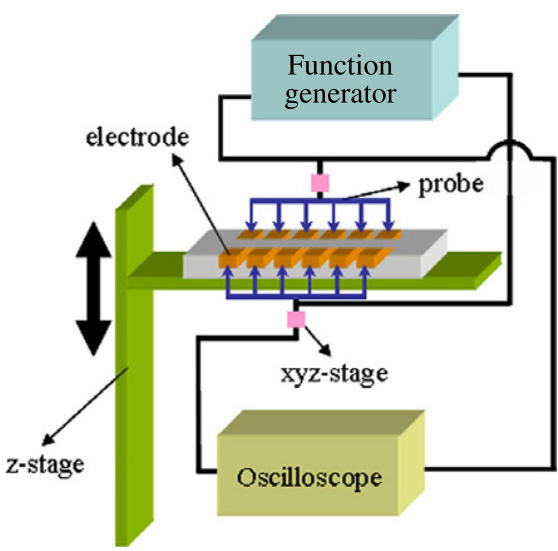

Figure 3. Schematic diagram of the apparatus.

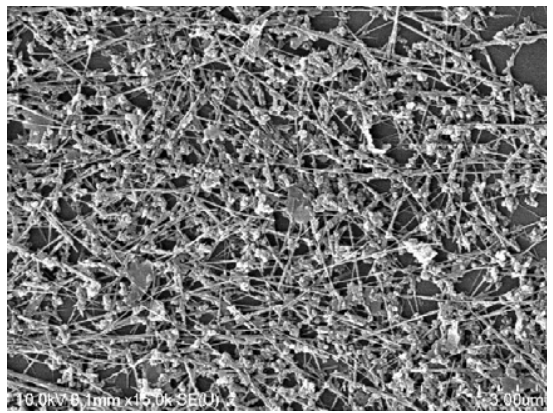

Figure 4. FESEM image of raw MWNTs.

\subsection{Apparatus}

2.2.1. Fabrication of purifying device. The purifying device used in the experiment is shown in figure 2. It is composed of a set of microelectrodes with a gap of $30 \mu \mathrm{m}$ in a $200 \mu \mathrm{m}$ microliquid channel. Twelve symmetric gold electrodes were placed on a silicon oxide plate, and the plate was covered with SU-8 with a thickness of $100 \mu \mathrm{m}$, having the shape of the microliquid channel. The entire fabrication process of the purifying device was achieved by a simple photolithography method. The ends of the purifying device are termed the outlet and inlet. Multi-walled carbon nanotube (MWNT) solution could flow along the channel from the inlet to outlet by capillary force.

2.2.2. Experimental set-up. Figure 3 shows a schematic diagram of the experimental apparatus for purifying the MWNTs. The apparatus consists of two elements: an approaching element, and an inducing element. The approaching element is composed of 12 probes with gold electrodes on a purifying device as counter electrodes. To support the purifying device, a holder was fixed to the translation stage of the $z$-axis. The 12 probes apply the voltage and frequency to each electrode on the purifying device through a conventional function generator, and each probe can be adjusted using an $x y z$-stage.

The inducing element of the apparatus is composed of a signal generator and a signal detector. An oscilloscope was used as the signal detector. The electric field can be generated 

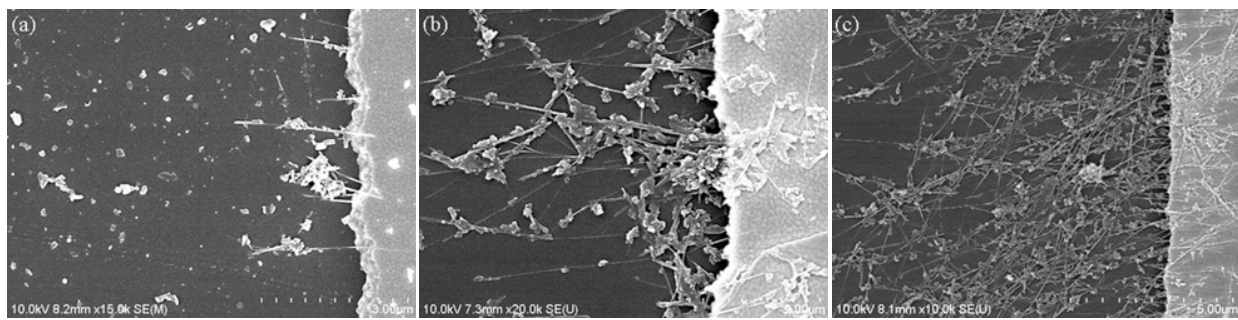

Figure 5. FESEM images of MWNTs attached to electrodes at a fixed frequency of $100 \mathrm{kHz}$ and voltages of (a) $0.1 V_{\mathrm{rms}} \mu \mathrm{m}^{-1}$, (b) $0.2 V_{\text {rms }} \mu \mathrm{m}^{-1}$ and (c) $0.3 V_{\text {rms }} \mu \mathrm{m}^{-1}$.
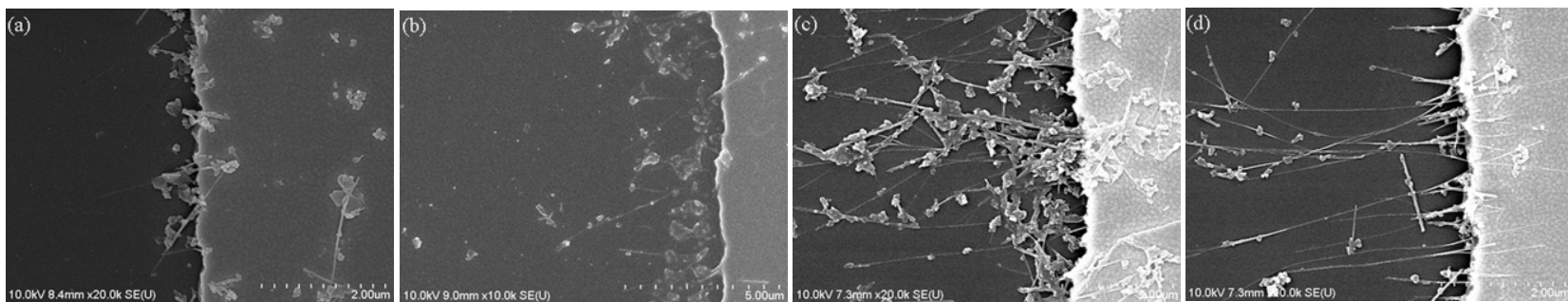

Figure 6. FESEM images of MWNTs attached to electrodes at a fixed voltage of $0.2 V_{\mathrm{rms}} \mu \mathrm{m}^{-1}$ and frequencies of (a) $0.01 \mathrm{kHz}$, (b) $1 \mathrm{kHz}$, (c) $100 \mathrm{kHz}$ and (d) $1 \mathrm{MHz}$

by an $\mathrm{AC}$ voltage, and it is possible to check the signal with the oscilloscope.

\section{Experimental section}

\subsection{Pre-treatment of the MWNTs}

MWNTs were obtained from Iljin Nanotech ${ }^{\circledR}$. These MWNTs were synthesized by the arc-discharge method. They had a diameter of $30 \mathrm{~nm}$, and a length of $5-10 \mu \mathrm{m}$. Figure 4 shows an FESEM image of the MWNTs in the raw state. As shown in this image, many carbon impurities are located around the MWNTs. These MWNTs had been dispersed in water with sodium dodecyl sulphate (SDS); consequently, this polymer structure obscured the FESEM image. Hence, the SDS was removed using acetone, and the MWNT solution was sonicated for $5 \mathrm{~min}$ at $70 \mathrm{~W}$ and $45 \mathrm{kHz}$ after dispersing the MWNTs in chloroform $\left(\mathrm{CHCl}_{3}\right)$ and isopropanol (IPA) at a 1:1 ratio. The concentration of the MWNT solution was fixed at $5 \mu \mathrm{g} \mathrm{ml}^{-1}$, and the volume of the droplets was fixed at $4 \mu \mathrm{l}$ based on consideration of the inlet volume.

\subsection{Determination of the purifying condition}

We supplied only an AC voltage to the purifying device. Through previous research, the approximate range of conditions under which CNTs attach and align well to the electrode was determined, i.e., a voltage of $0.3-1.3 V_{\mathrm{rms}} \mu \mathrm{m}^{-1}$, and a frequency of $0.1-20 \mathrm{MHz}$ [15-17]. Hence, it is necessary to avoid these conditions to purify the MWNTs, as the goal is to attach the carbon impurities to the electrodes rather than the MWNTs. First, we varied the voltage in a range of $0.1-0.3$ $V_{\mathrm{rms}} \mu \mathrm{m}^{-1}$, while using a fixed frequency of $100 \mathrm{kHz}$ in order to assess the influence of the voltage amplitude. On the basis of previously obtained experimental data, we subsequently varied the frequency in a range of $0.01-100 \mathrm{kHz}$ under a fixed voltage. The range of purifying conditions, with the appropriate voltage and frequency, was then narrowed on the basis of the previously obtained experimental data.

\subsection{Verification of the degree of purity}

We added a pair of extra electrodes with a gap of $20 \mu \mathrm{m}$ to the outlet of the purifying device, and applied a voltage of $0.8 V_{\text {rms }} \mu \mathrm{m}^{-1}$, and a frequency of $5 \mathrm{MHz}$ [15-17] as the purified MWNT solution passed by these electrodes. The above conditions, which were applied by another function generator, were chosen for the attachment and alignment of the MWNTs. Hence, the degree of purity could be verified based on an assessment of the difference between images taken before and after purification of the MWNTs attached and aligned on the extra electrodes added to the end of the outlet. However, these images only reveal verification of purification for a local area. For this reason, the experimental results were analysed with Raman spectroscopy in order to check the degree of purity from a qualitative point of view, over a macroscopic area.

\section{Results and discussion}

The electrostatic force is increased as the applied voltage is increased. As shown in figure 5, more MWNTs and carbon impurities were attracted to the electrodes as the voltage increased. Through iterations of the experiment, we observed that not only carbon impurities but also many MWNTs were attracted to the electrodes at voltages greater than $0.2 V_{\mathrm{rms}} \mu \mathrm{m}^{-1}$. However, as the goal of the study was to filter carbon impurities with the electrodes rather than MWNTs, a maximum voltage was determined to be $0.2 V_{\mathrm{rms}} \mu \mathrm{m}^{-1}$.

It was also found that more MWNTs relative to the quantity of carbon impurities attached and aligned to the 
(a)

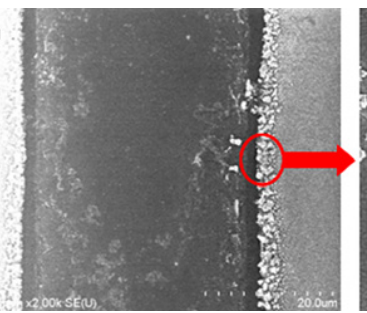

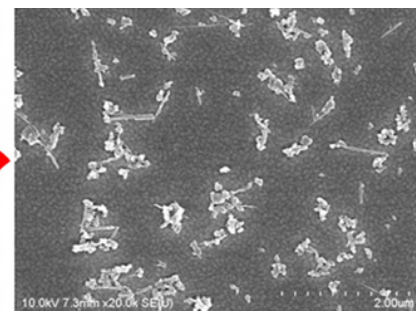
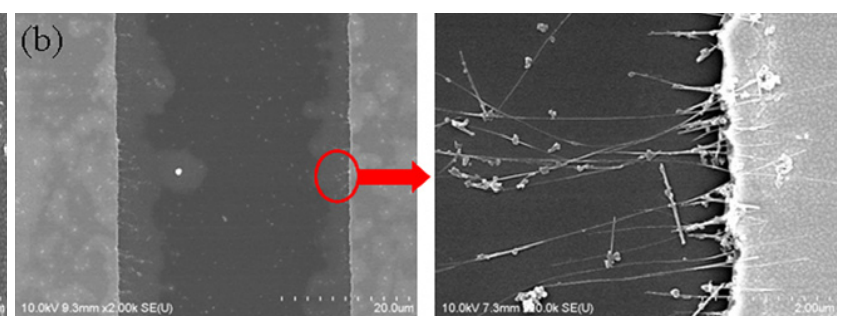

Figure 7. FESEM images of the area near the microelectrodes at a fixed voltage of $0.2 V_{\mathrm{rms}} \mu \mathrm{m}^{-1}$ and frequencies of (a) $1 \mathrm{kHz}$, (b) $1 \mathrm{MHz}$.
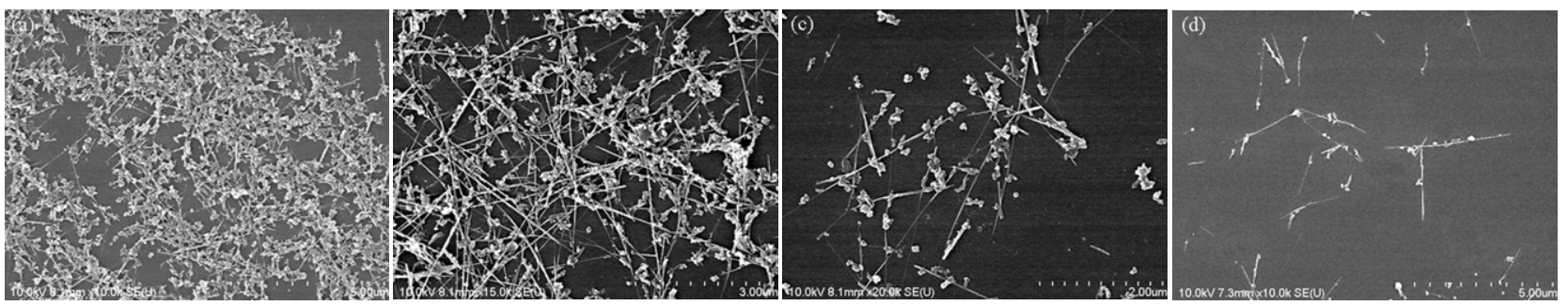

Figure 8. FESEM images of MWNTs at the outlet area under a fixed frequency of $100 \mathrm{kHz}$ and voltages of (a) $0.00 V_{\mathrm{rms}} \mu \mathrm{m}^{-1}$, (b) $0.10 V_{\text {rms }} \mu \mathrm{m}^{-1}$, (c) $0.15 V_{\text {rms }} \mu \mathrm{m}^{-1}$ and (d) $0.20 V_{\text {rms }} \mu \mathrm{m}^{-1}$.
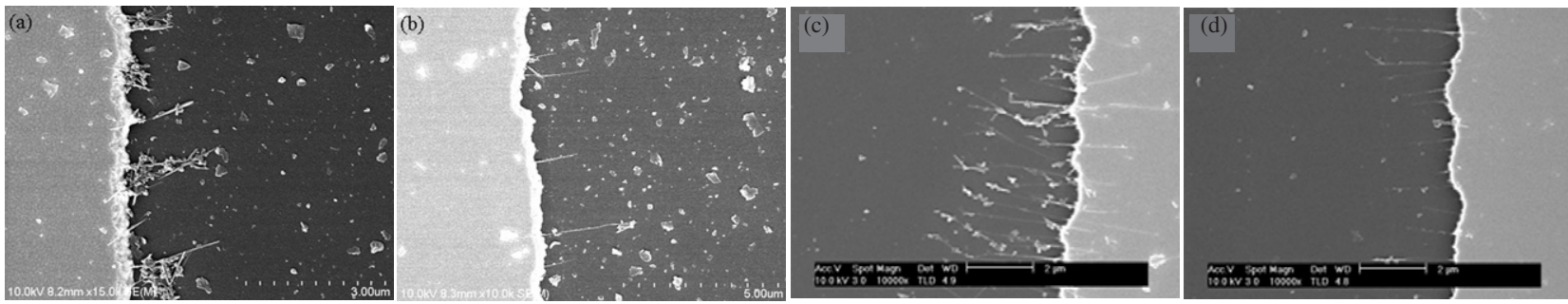

Figure 9. FESEM images of the MWNTs at the extra electrodes, (a) and (c) before purification, (b) and (d) after purification.

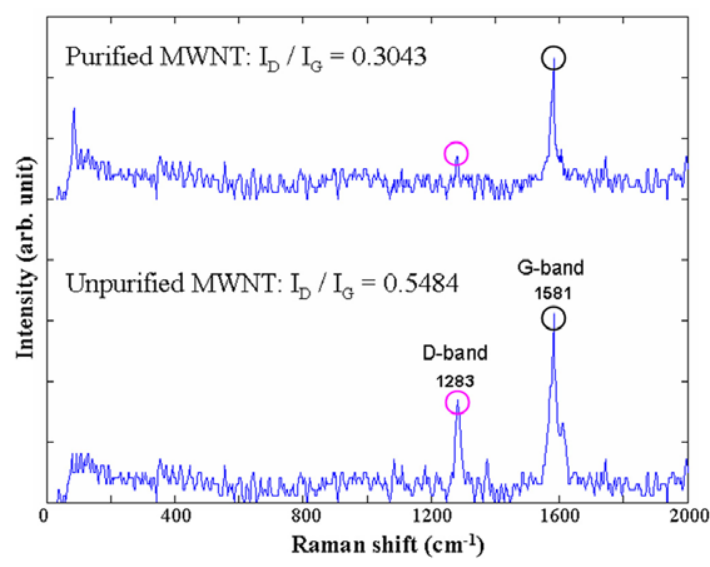

Figure 10. Raman spectra $\left(\lambda_{\mathrm{exc}}=1064 \mathrm{~nm}\right)$ of the two MWNT materials.

electrodes at a frequency of more than $100 \mathrm{kHz}$, as shown in figure 6. The quantity of attached carbon impurities also decreased at a frequency of less than $100 \mathrm{kHz}$. In other words, the quantity of carbon impurities attached to the electrodes decreased at a frequency lower than or higher than $100 \mathrm{kHz}$, indicating that the quantity of carbon impurities attached to the microelectrodes is dependent upon the frequency at a fixed proper amplitude of voltage. As shown in figure 7, additional carbon impurities were filtered at a frequency of $1 \mathrm{kHz}$ as compared to the state at a frequency of $1 \mathrm{MHz}$. This result can be explained by the following phenomenon. Carbon particles are in a colloidal state in a mixture of IPA and $\mathrm{CHCl}_{3}$, and are positively charged and covered with negative ions. Negative ions slip off positively charged particles at a low frequency of less than $100 \mathrm{kHz}$, and therefore carbon impurities can move along the electric field line. However, at a high frequency of more than or much less than $100 \mathrm{kHz}$, carbon impurities do not readily move along the field line, because they are charged particles covered with negative ions [18]. MWNTs can be attracted to the electrodes in a nonuniform electric field, which can be induced by imperfectly symmetric electrodes or by the irregular interface shape of the electrodes on a microscale. As shown in figure 8, the MWNTs could be purified with additional voltage, but they are then reduced in quantity at the outlet area, as a result of the attachment of MWNTs. However, most MWNTs flowed through the apparatus without attaching to the electrodes, along the microliquid channel at a voltage of $0.2 V_{\mathrm{rms}} \mu \mathrm{m}^{-1}$, and a frequency of $1 \mathrm{kHz}$.

In order to verify the difference between the MWNTs before and after purification, we aligned and attached them, before and after purification, to the extra electrodes, which 

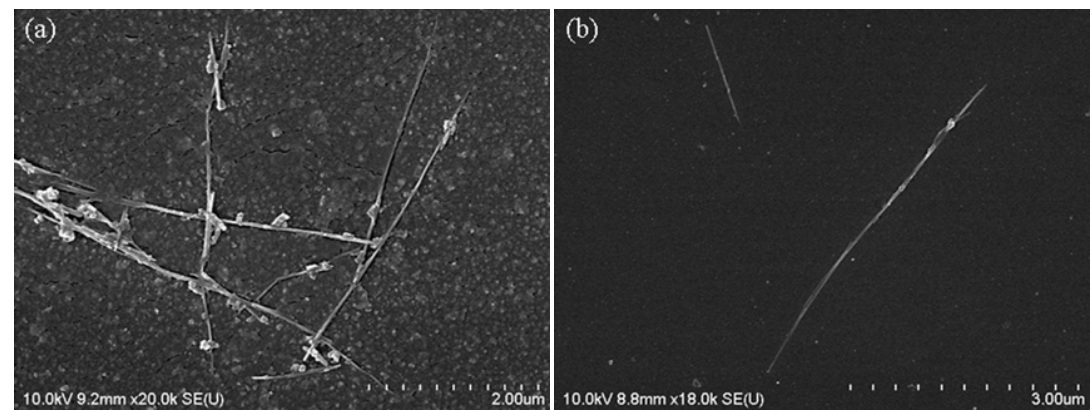

Figure 11. FESEM image of MWNTs at the (a) inlet and (b) outlet of the purifying device at a voltage of $0.2 V_{\mathrm{rms}} \mu \mathrm{m}^{-1}$ and a frequency of $1 \mathrm{kHz}$.

were added to the outlet of the purifying device. The added electrodes had a gap size of $20 \mu \mathrm{m}$. Essentially, the gap size of the microelectrodes on the purifying device was designed considering the length of the MWNTs. In other words, the maximum length of the samples in the experiment is approximately $10 \mu \mathrm{m}$, thus it was necessary to design a gap size of longer than $10 \mu \mathrm{m}$, so that the samples would flow smoothly. Moreover, in this report, the applied voltage indicates the amplitude per gap size of the microelectrodes, rather than the amplitude of the voltage. Therefore, it was also necessary to consider the maximum output voltage level of the function generator when the gap size of the microelectrodes was designed. The function generator used in this experiment has a maximum output voltage level of nearly $18 V_{\text {rms }}$, hence the microelectrodes were designed with a gap size of $30 \mu \mathrm{m}$ in the purifying device, not only for a smooth sample flow but also to give a surplus of applied voltage in a parametric study. However, in the design of the extra microelectrodes, it was not necessary to consider the length or the flow of the MWNTs. Instead, it was essential to design the extra microelectrodes with a properly sized gap which was based on consideration of the amplitude of the applied voltage, as the maximum output voltage of the function generator was approximately $18 V_{\mathrm{rms}}$. Owing to the fact that it was necessary to apply $0.8 V_{\mathrm{rms}} \mu \mathrm{m}^{-1}$ to the output region in the purifying device, the extra microelectrodes were designed with a gap size of $20 \mu \mathrm{m}$.

As shown in figure 9, there is clear a difference between the MWNTs before and after purification. When the MWNTs purified through an electric field generated by a voltage of $0.2 V_{\text {rms }} \mu \mathrm{m}^{-1}$, and a frequency of $1 \mathrm{kHz}$, were aligned and attached to extra electrodes, virtually no carbon impurities were found near the MWNTs. However, the carbon impurities still exist near the MWNTs in the case where the electric field is not generated when MWNTs passed along the microliquid channel. It is believed that a number of large particles near the extra microelectrode in figures 9 (a) and (b) are merely dirt, and not carbon impurities, which may be due to an incomplete cleaning process after fabrication of the purifying device. There were virtually no particles, as explained in the paragraph above and shown in figures 9(c) and (d), when this experiment was conducted with another purifying device. However, small quantities of carbon impurities remain near the MWNTs, although these MWNTs were able to pass along the microliquid channel under a voltage of $0.2 V_{\mathrm{rms}} \mu \mathrm{m}^{-1}$, and a frequency of $1 \mathrm{kHz}$. This problem comes from an incomplete dispersion state of the MWNT sample. In other words, separation between the carbon impurities and the MWNTs is needed to effectively filter the carbon impurities before the sample starts flowing. However, it is hard to achieve a complete separation between carbon impurities and MWNTs due to the functional limit of this purifying device. For this reason, an experimental result, as shown in figures 9 (b) and (d), could not be obtained in three or four cases out of ten. This can be resolved if the inlet part of the purifying device is modified. For instance, if a micro-mixer or vibrator was added to the inlet of the purifying device, good individualization or dispersion could be possible in samples, and additional carbon impurities could be eliminated by the electric field, as compared to the samples in figures 9(b) and (d).

We used Raman spectroscopy to evaluate the electricfield-assisted purification of MWNT samples from a qualitative point of view. Room temperature Raman spectra of the samples were obtained using a Brukeopitcs ${ }^{\circledR}$ RFS 100/S FT (Fourier transform) Raman spectrometer. The Raman spectra using the $\lambda=1064 \mathrm{~nm}$ excitation line are shown in figure 10 . These spectra are considered to be typical of MWNTs at $\lambda=$ $1064 \mathrm{~nm}$ [19]. The two spectra correspond to the purified and the nonpurified samples shown in the FESEM images in figure 11. The two different regions of interest are in the $\mathrm{G}$ band region near $1580 \mathrm{~cm}^{-1}$, and in the $\mathrm{D}$ band near $1280 \mathrm{~cm}^{-1}$. As shown in figure 10, in the purified sample, the $\mathrm{D}$ band is weaker and the $\mathrm{G}$ band is narrower. A direct measurement of the $\mathrm{D}$ band to $\mathrm{G}$ band intensity ratio gives $I_{\mathrm{D}} / I_{\mathrm{G}}=0.3043$ after purification, as compared to $I_{\mathrm{D}} / I_{\mathrm{G}}=$ 0.5484 before. This reduction by $44.5 \%$ of $I_{\mathrm{D}} / I_{\mathrm{G}}$ is believed to be related to the removal of most of the carbon particles or of the amorphous carbon [20]. Therefore, the entire experimental results show that this purifying device can be used not only for purification of MWNTs but also in the fabrication of impurityfree MWNTs.

\section{Acknowledgment}

The authors would like to express their appreciation to the Brain Korea 21 project in 2006 for partial financial support of this work.

\section{References}

[1] Iijima S 1991 Nature 354 56-8

[2] Lee S M and Lee Y H 2000 Appl. Phys. Lett. 76 2877-9 
[3] Collins P G, Bradley K, Ishigami M and Zettl A 2000 Science 287 1801-4

[4] Deheer W A, Chatelain A and Ugarte D 1995 Science 270 1179-80

[5] Journet C et al 1997 Nature 388 756-8

[6] Ko F-H, Lee C-Y, Ko C-J and Chu T-C 2005 Carbon 43727

[7] Rinzler A G et al 1998 Appl. Phys. A 67 29-37

[8] Ebbesen T W, Ajayan P M, Hiura H and Tanigaki K 1994 Nature 367519

[9] Hiura H, Ebbesen T W and Tanigaki K 1995 Adv. Mater. 7275

[10] Dujardin E, Ebbesen T W, Krishnan A and Treacy M J 1998 Adv. Mater. 10 611-3

[11] Bandow S, Rao A M, Williams K A, Thess A, Smalley R E and Eklund P C J 1997 Phys. Chem. B 101 8839-42

[12] Shelimov K B, Esenaliev R O, Rinzler A G, Huffman C B and Smalley R E 1998 Chem. Phys. Lett. 282 429-34
[13] Murphy R et al 2002 J. Phys. Chem. B 106 3087-91

[14] Pohl H A 1978 Dielectophoresis (Cambridge: Cambridge University Press)

[15] Lee H W 2005 A Study on Alignment and Attachment of Carbon Nanotube Using Electric Field (Daejeon: Korea Advanced Institute of Science and Technology Press)

[16] Lee H W, Kim S H, Kwak Y K, Lee E S and Han C S 2005 Sens. Actuators A 125 41-9

[17] Lee H W, Kim S H, Kwak Y K and Han C S 2005 Rev. Sci. Instrum. 76046108

[18] Yamamoto K, Akita S and Nakayama Y 1996 Japan. J. Appl. Phys. 35 L917-8

[19] Lee Y H 2005 Sae Mulli (The Korean Physical Society) $5184-114$

[20] Benoit J M, Buisson J P, Chauvet O, Godon C and Lefrant S 2002 Phys. Rev. B 66073417 